Case Report

\title{
Collecting duct carcinoma: A rare entity
}

Ferdinant Martinus Djawa ${ }^{1,2}$, Anny Setijo Rahaju ${ }^{1 *}$

1) Departement of Pathological Anatomic, Faculty of Medicine, Universitas Airlangga/ Dr. Soetomo General Academic Hospital, Surabaya, Indonesia

2) Faculty of Medicine, Universitas Cenderawasih, Jayapura, Indonesia

A R T I C L E I N F O

\begin{tabular}{ll}
\hline Submitted & : July 2019 \\
Accepted & : September 2019 \\
Published & : January 2020
\end{tabular}

\section{Keywords:}

renal cell carcinoma, collecting duct carcinoma, urothelial carcinoma, PAX8, p63

\section{*Correspondence:}

anny_sr@fk.unair.ac.id

\section{ABSTRACT}

Collecting duct carcinoma is a rare and highly aggressive subtype of renal cell carcinoma. The incidence rate is less than $1-2 \%$ of all renal tumors and usually, affect middleaged adult, commonly in men. We reported a 76-year-old man complains of an intermittent painless gross hematuria, abdominal mass and left flank pain for approximately three months. The CT abdomen showed a slightly enhancing solid mass in the left kidney and para-aorta lymphadenopathy. Cut surfaces of the kidney showed a solid-cystic and illdefined greyish-white tumor. Microscopically, tumor formed solid sheets and tubulopapillary structures lined by neoplastic cells, hobnailing nuclei, abnormal mitotic, and a desmoplastic stroma with lymphoplasmacytic infiltration, and the immunochemical profile were PAX8 (+) /p63 (-). Based on these findings, the diagnosis was a collecting duct carcinoma. This tumor arising from the collecting duct of Bellini in the renal medulla, accounts for less than 1-2\% of all renal masses and important to be distinguished from other tumors due to differences in prognosis and therapeutic. Histopathological examination is needed to establish the diagnosis. A case of collecting duct carcinoma that occurred in a 76-year-old man has been reported. A definitive diagnosis can only be done with a detailed histopathological examination for patient management benefits. 


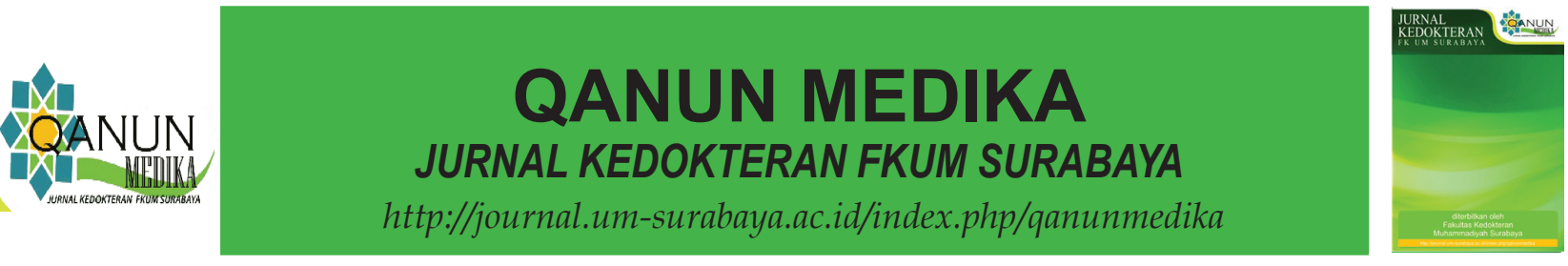

\section{INTRODUCTION}

Collecting duct carcinoma (CDC) of the kidney is a rare subtype of the renal epithelial cell carcinomas (RCCs), arising from the distal segment of collecting ducts of Bellini, usually located in the middle of renal sinus region. It accounts for less than $1-2 \%$ of all renal masses but highly aggressive, and has a poor prognosis. They can affect the age range 13-83 years old with a mean age of 55 years, and there is a 2:1 male predominance. Due to its rarity, the tumor is still limited understood (Ciszewski, Jakimów, \& Smolska-Ciszewska, 2015; Albadine et al., 2010; Harbin, Styskel, Patel, Wang, \&Eun, 2015).

In these past two decades, paradigms that developed in the effort to classify pathological morphologic subtypes are aimed at the better understanding molecular origin, clinical behavior, similarities, and differences between RCC variants with the hope providing clinical and therapeutic opportunities to improve patient care (Shuch et al., 2015).

\section{CASE REPORT}

A 76-year-old man experiences intermittent painless gross hematuria for three months. The patient had a recent onset left flank intermittent pain since one-month before admitted, also a history of gradual weight loss and decreased appetite for two months. A mobile palpable mass in the upper left abdominal quadrant, measuring $7 \times 5 \mathrm{~cm}$, was found during the physical examination. CT-scan with contrast at abdomen showed a slightly enhancing solid mass in the left kidney, measuring $7 \times 6 \times 6 \mathrm{~cm}$, and para-aorta lymphadenopathy, $3.4 \times 4.2 \mathrm{~cm}$ in size. Other clinical and laboratory findings were within normal limits. The patient underwent the left radical nephrectomy.

The resected left kidney was enlarged, measured $10 \times 7.3 \times 5 \mathrm{~cm}$. On gross examination, the renal surface was smooth and adherent to perinephric fat. The cut surface showed an illdefined greyish-white tumor, firm in consistency, solid with a few cystic areas, measuring 7x6x6 $\mathrm{cm}$, which located in the middle and lower poles predominantly involving the medulla, with minimal hemorrhages or necrosis. The tumor was seen invading the capsule and extending into the perinephric fat. The hilus was uninvolved.

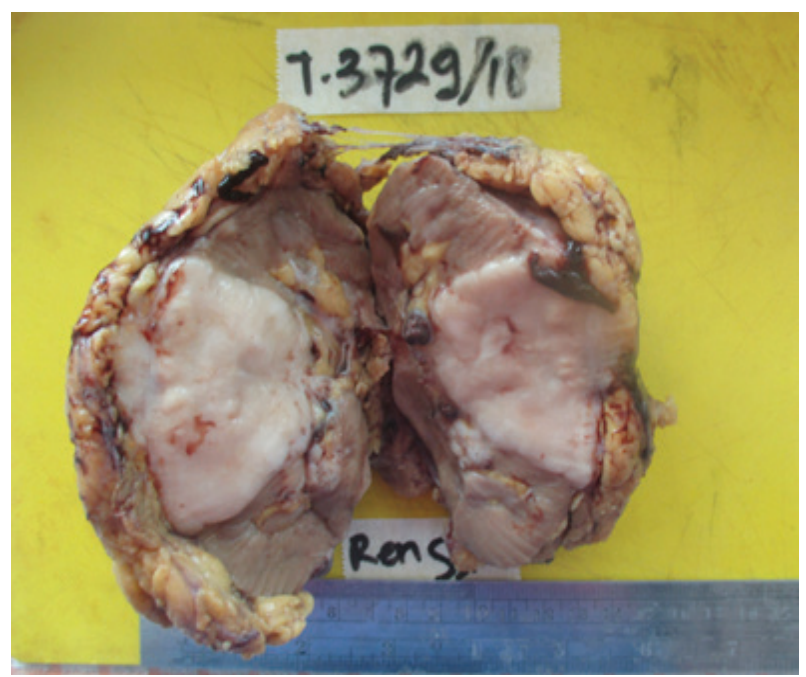

Figure 1. The rejected left kidney. Cut section showed an ill-defined greyish-white tumor (white arrow),

solid-cystic (arrows head) minimal hemorrhages, measuring $7 \times 6 \times 6 \mathrm{~cm}$, located in the renal medulla. Renal surface smooth covered by fat ( yellow arrow ). The tumor invades to perinephric fat (blue arrow). (Source: Author, 2018)

Microscopically, the tumor formed solid sheets and tubulopapillary structures lined by proliferation of round to polygonal neoplastic cells with pleomorphic vesicular nuclei, prominent nucleoli, and eosinophilic cytoplasm. Many abnormal mitotic figures were noted. The intervening stroma was desmoplastic with moderate lymphoplasmacytic infiltration. A few tubules were also seen lined by atypical cuboidal cells with evidence of hobnail nuclei. The tumor cells showed nuclear expression of PAX8 but were stained negatively for p63. 

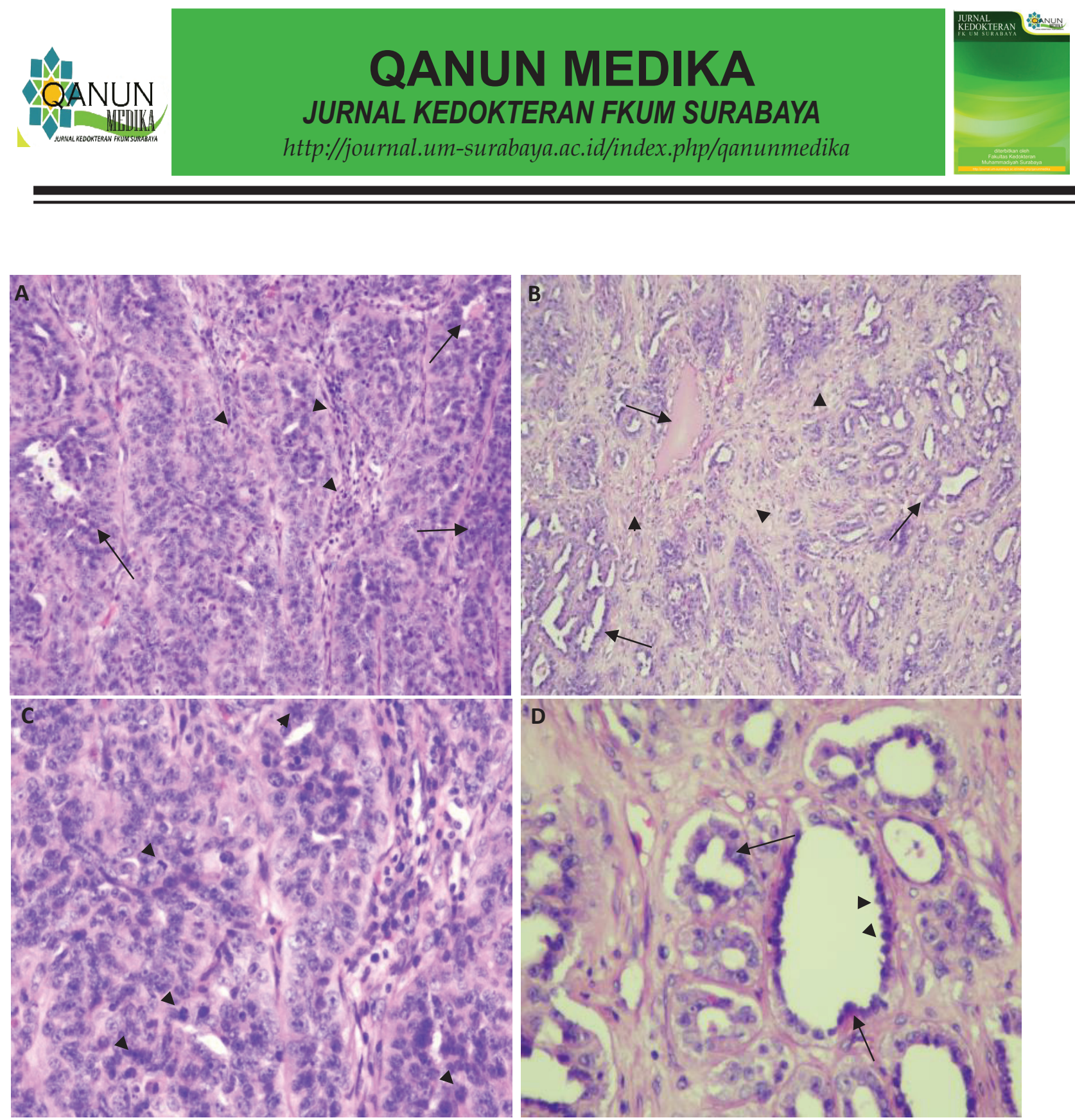

Figure 2. Histologically. A. Formation of solid sheets (arrows), infiltration into the fibrous stroma that infiltration by lymphoplasmacytic cells (head arrows, x20), B. Predominantly tubulopapillary structures (arrows), sometimes content material like mucin, with reactive stromal desmoplastic (arrowhead, x20), C. A few of abnormal mitotic figure (head arrows) (x20), D. Tubules lined by high-grade eosinophilic, basophilic cells (arrows) with hob-nail nuclei features (arrows head, x40). (Source: Author, 2018)
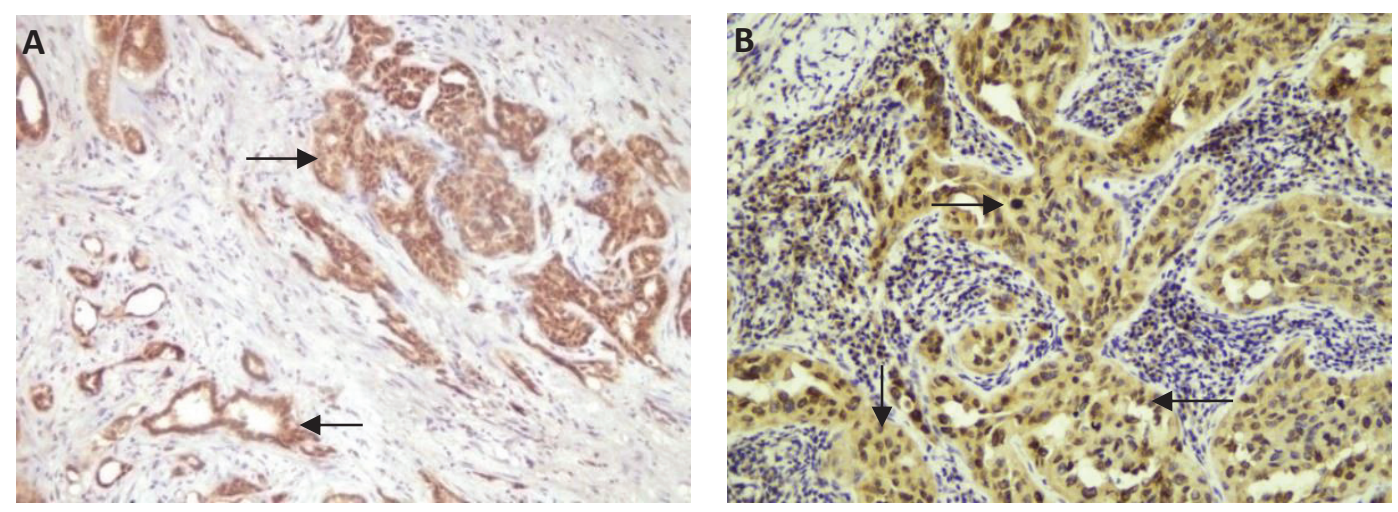


\section{QANUN MEDIKA Vol 4 No 1 JANUARY 2020}
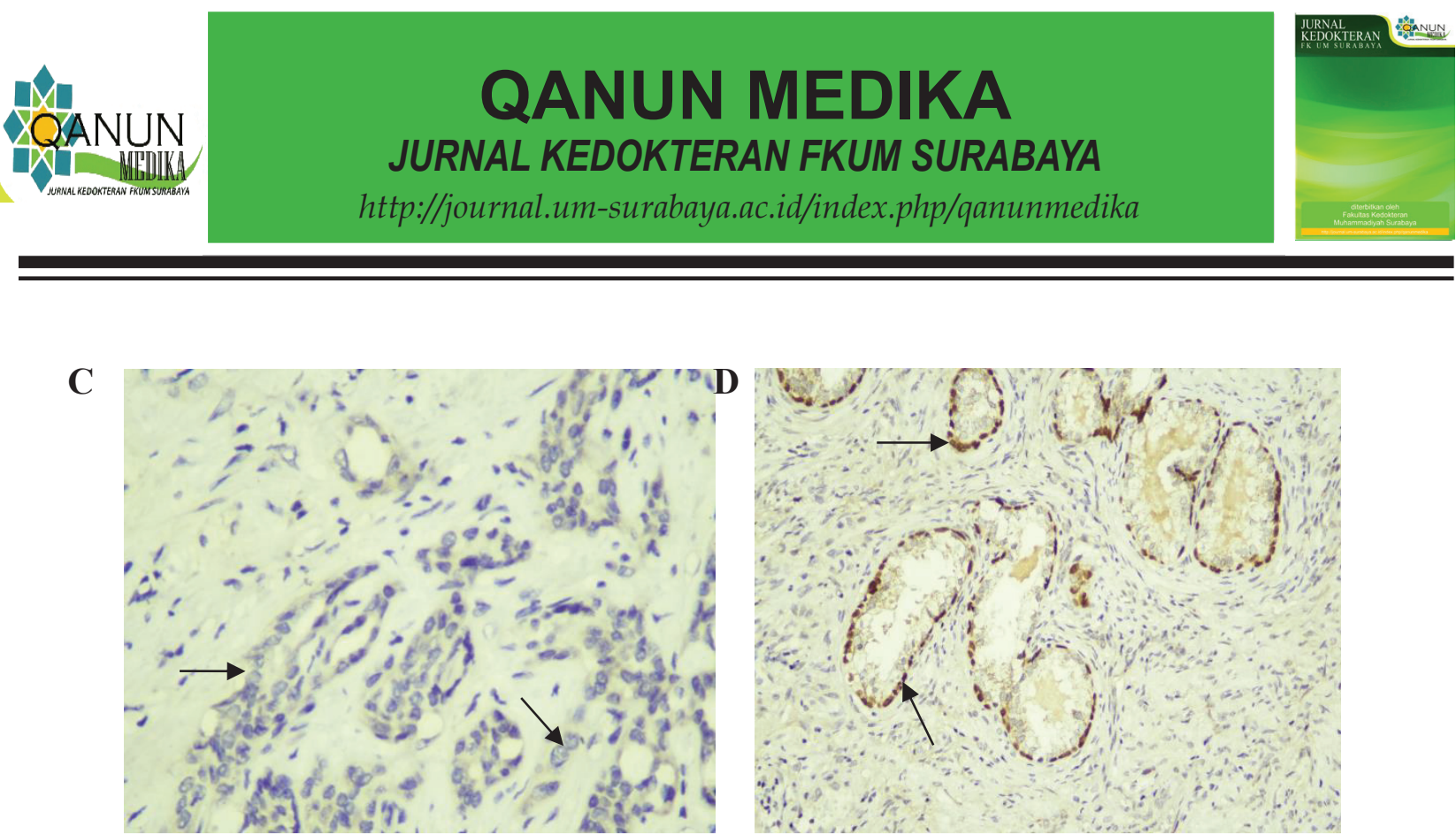

Figure 3. Immunohistochemically. A. The clusters of tumor cells showed expresses PAX8 (arrows, x20), B. Positif stained for PAX8 at nuclei of tumor cells (arrows, x40) C. Nuclei of tumor cells not express p63 (arrows, x40) D. p63 control: showed positive stained at basal cells nuclei of the ducts (arrows, x20) (Source: Author, 2019)

\section{DISCUSSION}

Many factors are believed to be related to the risk of kidney cell carcinoma such as demographics, smoking, the use of fenacetin, obesity, lack of physical activity, exposure to industrial or environmental agents, hypertension, hyperglycemia and hypertriglyceridemia, and also blood concentrations of vitamin D-binding protein (VDBP). The risk of RCC increases in obesity, approximately $20-35 \%$ higher for every $5 \mathrm{~kg} / \mathrm{m} 2$ of higher BMI (Petejova \& Martinek, 2016).

$\mathrm{CDC}$ is defined as a malignant epithelial tumor arising from the lining cells of the collecting ducts of Bellini in the renal medulla. This is different from most RCCs, which occur due to malignant proliferation of the proximal tubular of nephron cells, and from urothelial carcinoma (UC) which arises from the transitional epithelium lining of the mucosa of the bladder, renal pelvis and ureters (Mishra, Manikandan, Dorairajan, Mittal, \& Rekha, 2016).

Fleming and Lewi first introduced CDC in 1986 as a new entity that differs from other subtypes of renal cell cancer. The study by Pepek and colleagues found that the incidence rate was only $1-2 \%$ of all renal tumors, generally affected people ages $13-83$ years with an average age of 55 years. The male to female incidence ratio is 2:1 and found mostly in white patients, African-American, and some other races (Seo, Yoon, \& Ro, 2017; Harbin et al., 2015; Mishra et al., 2016).

Similar to our case, most of patients complain some symptoms such as hematuria, flank pain, palpable abdominal mass or distant metastases, weight loss, but some cases might be asymptomatic (Harbin et al., 2015; Liu J, Li Y, Su Z, Duqun Chen, Ni L, Mao X, 2016; Albadine et al., 2010). Usually, polycythemia (erythrocytosis) in patients with RCC is caused by the production of ectopic erythropoietin by cancer cells. Symptoms such as fever, weight loss, and general fatigue in many malignancies, are thought to be mediated by cytokines, especially TNF $\alpha$ and IL-6 (Petejova\&Martinek, 2016). More than $30 \%$ of CDC patients present with metastasis at the time of diagnosis, most commonly in regional lymph nodes (about 80\% of cases), lung, adrenal gland, and liver, and about $60-70 \%$ of them die within a two-year period (Muglia \&Prando, 2015; Dall'Oglio et al., 2008; Wu, Zhu, Zhu, Chen, \& Wang, 2015).

The literature mentioned that on the CT-Scan 
examination, the tumor is usually located in the medullary, with weak enhancement (hypovascular pattern), renal sinus involvement and infiltrative growth pattern. It usually still maintains the renal contour and has cystic components. The para-aortic lymphadenopathy is also indicated (Mishra et al., 2016). The hyperdense appearance of tumors on unenhanced CT refers to a minimal intratumor hemorrhage (haemosiderin deposition) (Wu et al., 2015). These findings were also found in our case.

In this case, macroscopically, tumors are generally firm, gray-white, with poor margins but without extensive necrosis or hemorrhage. The tumor invasion into perirenal fat was found. The presence of tubular or tubulopapillary infiltrativepatterns, intense stromal desmoplasia, and infiltration of chronic inflammatory cells in and around tumors, help to establish a definitive diagnosis. The tumor cells usually moderate to severe eosinophilic, basophilic, or amphophilic nuclear pleomorphism and mitosis, with hobnails nuclei feature. Lymphovascular invasion can be seen, and metastases can occur in regional lymph nodes, bones, adrenal glands, lungs, and skin (Konjengbam \& Singh, 2017; López, Larrinaga, Kuroda, \& Angulo, 2015). The other patterns include a variable admixture of solid cords, sheets, papillary formations, and cystic dilated spaces. The tubules, glands, or cysts showed focal to prominent intra-luminal basophilic to amphophilic mucin in other cases (Gupta et al., 2012).

The guidelines for diagnosing CDC are based on the criteria established by the International Society of Urological Pathology (ISUP) on the conference of renal neoplasia in Vancouver 2013, and the WHO classification 2016, as follows: (1) at least a portion of tumors involving the medullary region, (2) formation of dominant tubules, (3) desmoplastic stromal reaction, (4) high-grade features, (5) infiltrative growth patterns, (6) and no other RCC subtypes or urothelial carcinomas were found (Seo et al., 2017; Harbin et al., 2015).

CDC needs to be distinguished from other renal neoplasms such as papillary renal cell carcinoma, renal medullary carcinoma, and high-grade urothelial carcinoma, also the possibility of metastatic carcinoma, especially from GIT. Papillary RCCs usually shows a well-demarcated lesion on a cut surface, yellow or brown color, and lack of stromal desmoplasia on the histological feature. Immunohistochemically, papillary renal cell carcinoma shows frequent positivity for CK7 and, occasionally, CD9 (Konjengbam\& Singh, 2017). Renal medullary carcinoma commonly shows islands of anastomosing tubule, and cords forming irregular microcystic spaces and usually occur in young black patients with sickle cell trait, with a different history and age of patients, and the INI-1 retention is strictly able to rule out the possibility of medullary carcinoma (Konjengbam \& Singh, 2017; Zheng et al., 2017; Gupta et al., 2012).

Although the CDC behavior and immunohistochemical properties are similar to urothelial carcinoma (UC), both have a distinct genetic pattern. The cytogenetic profiles of the CDC are associated with the chromosomal DNA losses at 8p, 16p, 1p, and 9p, and gains at 13q (Shuch et al., 2015; Harbin et al., 2015; Petejova \& Martinek, 2016). Both macroscopically and microscopically, the invasive UC growth pattern gives an appearance similar to the CDC. The UC mostly forms nests and cell cords, pseudo-glandular patterns, and squamous metaplasia, and in particular, shows intraepithelial neoplasia in the pelvic mucosa, which is not found in CDC cases (Kobayashi et al., 2008). As the CDC tumor cells spread in the cortical collecting tubule, the lesion develops characteristics similar to those of upper tract urothelial carcinoma (Jung, Oh, \& Shin, 2017). The high-grade UC does not express p63 and GATA-3 (Konjengbam\& Singh, 2017; Zheng 


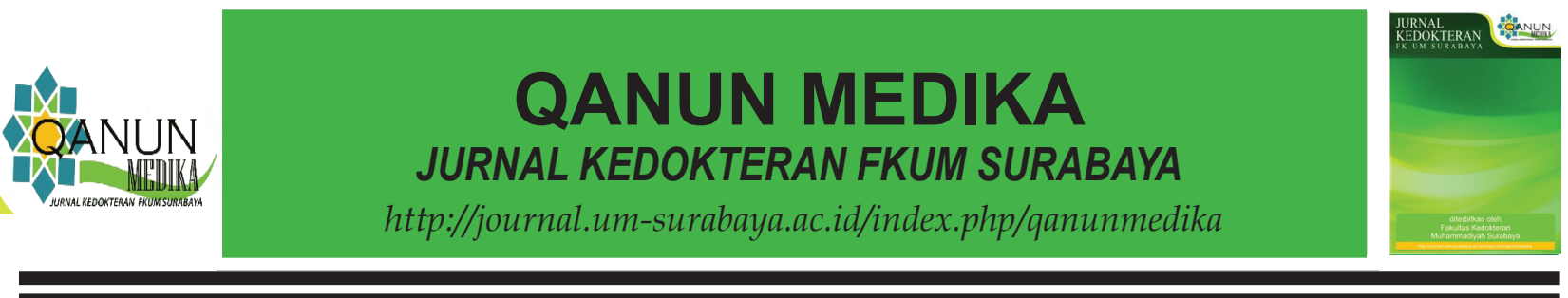

et al., 2017) Most CDC tumor cells express PAX8, High Molecular Weight Cytokeratin, sometimes also CK7, and strongly positive for epithelial membrane antigen (EMA), focal reactive with vimentin, but they do not express CK20, CD117, CD10, GATA3 and p63 on immunohistochemistry staining ( $\mathrm{Hu}$ et al., 2015; López et al., 2015; Zheng et al., 2017).

PAX8 is consistently positive towards the CDC, normal collecting ducts, and differentiating nephrons. Immunohistochemistry is particularly useful in the differential diagnosis, where panels of GATA3, p63, and PAX8 are good guidelines for differentiating the CDC from invasive urothelial carcinoma. When the tumor showing immune-profile PAX8 $(+) /$ p63(-) and/or GATA3 (-) are favor to CDC, while a profile of PAX8 (-)/p63 (+) and/or GATA3 $(+)$ tends to UC diagnosis (Seo et al., 2017; Harbin et al., 2015). A research by Albadine and colleagues found all 21 (100\%) CDCs were positive for PAX8, and p63 was positive in 3 of 21 (14\%) CDC cases (PAX8 $+/$ p63 +). In CDC lesions with urothelial differentiation, it can be observed that the tumor cells express p63 focally (Albadine et al., 2010)

As in our case, that immunohistochemical staining shows tumor cell positivity for PAX8 and negative for p63 (PAX8 + /p63 -), where this scenario has a specificity of $100 \%$ and a positive predictive value of $100 \%$ in the diagnosis of Collecting Duct Carcinoma (Jung et al., 2017).

Current treatment options for CDC are based on standards set for renal cell carcinoma, but due to their aggressiveness and also with metastasis, the intervention such as cytoreductive nephrectomy is less useful for the survival of this case and more suitable for the management of RCC cells (ccRCC), which is known resistant to systemic chemotherapy (Sui et al., 2017). Majority of CDC with metastases have a very poor prognosis, but pathological similarities with UC are the main reason to use sophisticated chemotherapy agents such as Gemcitabine plus Cisplatin; therefore CDC may be chemosensitive/radiosensitive (Sui et al., 2017; Kawaguchi, Ito, Shimazaki, \& Asano, 2017).

Pathological diagnosis is needed to establish a definitive diagnosis of the CDC, but imaging techniques can help to lead to early diagnosis (Harbin et al., 2015). To increase the survival rates, it is important to establish an early diagnosis, treatment, and surgery performed on patients with organ limited small tumors (Ciszewski et al., 2015).

\section{CONCLUSION}

A case of collecting duct carcinoma that occurred in a 76-year-old man has been reported. Collecting ductal carcinomas is a rare entity that must be distinguished from other renal neoplasms because of the differences in prognosis and therapy. A definitive diagnosis can only and must be done with a detailed histopathological examination for patient management benefit.

\section{REFERENCES}

Albadine, R., Schultz, L., Illei, P., Ertoy, D., Hicks, J., Sharma, R., ... Netto, G. J. (2010). PAX8 (+)/p63 (-) Immunostaining Pattern in Renal Collecting Duct Carcinoma (CDC). The American Journal of Surgical Pathology, 34(7), 965-969. https://doi.org/10.1097/ pas.0b013e3181dc5e8a

Ciszewski, S., Jakimów, A., \& SmolskaCiszewska, B. (2015). Collecting (Bellini) duct carcinoma: A clinical study of a rare tumor and review of the literature. Canadian Urological Association 


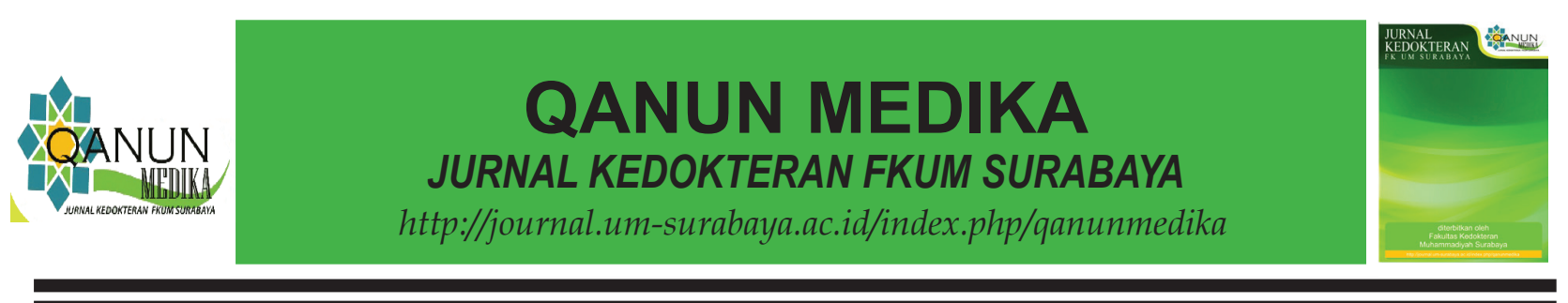

Journal, 9(9-10), 589. https://doi. org/10.5489/cuaj.2932

Dall'Oglio, M. F., Antunes, A. A., Pompeo, A. C., Mosconi, A., Leite, K. R. M., \& Srougi, M. (2008). Prognostic relevance of the histological subtype of renal cell carcinoma. International Braz J Urol, 34(1), 3-8. https://doi.org/10.1590/ S1677-55382008000100002

Gupta, R., Billis, A., Shah, R. B., Moch, H., Osunkoya, A. O., Jochum, W., ... Amin, M. B. (2012). Carcinoma of the Collecting Ducts of Bellini and Renal Medullary Carcinoma. The American Journal of Surgical Pathology, 36(9), 1265-1278. https://doi.org/10.1097/ pas.0b013e3182635954

Harbin, A. C., Styskel, B. A., Patel, V., Wang, H., \& Eun, D. D. (2015). Collecting Duct Renal Cell Carcinoma Found to Involve the Collecting System During Partial Nephrectomy: A Case Report. Journal of Kidney Cancer and VHL, 2(3), 134. https://doi.org/10.15586/jkcvhl.2015.37

Hu, Y., Zhou, H., Wang, G., Song, Z., Zhao, C., \& Wang, Y. (2015). Collecting duct carcinoma of the kidney: A case report. Oncology Letters, 9(6), 2902-2904. https://doi.org/10.3892/ol.2015.3085

Jung, H. J., Oh, H. K., \& Shin, H. S. (2017). Collecting Duct Carcinoma (Bellini Duct Carcinoma) Presented as a Renal Cyst. The Korean Journal of Urological Oncology, 15(3), 187-191. https://doi. org/10.22465/kjuo.2017.15.3.187

Kawaguchi, M., Ito, K., Shimazaki, H., \& Asano, T. (2017). A Case of Metastatic Collecting Duct Carcinoma Whose Massive Skull Bone Metastasis was Prominently Reossified by Gemcitabine Plus Cisplatin Chemotherapy Combined with Zoledronic Acid. Urology Case
Reports, 15, 14-16. https://doi. org/10.1016/j.eucr.2017.07.002

Kobayashi, N., Matsuzaki, O., Shirai, S., Aoki, I., Yao, M., \& Nagashima, Y. (2008). Collecting duct carcinoma of the kidney: an immunohistochemical evaluation of the use of antibodies for differential diagnosis. Human Pathology, 39(9), 1350-1359. https://doi.org/10.1016/j. humpath.2007.11.020

Konjengbam, L., \& Singh, T. (2017). Collecting duct carcinoma: Sarcomatoid variant - A rare entity. Journal of Medical Society, 31(1), 52. https://doi.org/10.4103/09724958.198466

Liu J, Li Y, Su Z, Duqun Chen, Ni L, Mao X, Y. S., and L. Y. (2016). SM Gr up SM Journal of Case Reports Sickness. 2(2), $1-2$.

López, J. I., Larrinaga, G., Kuroda, N., \& Angulo, J. C. (2015, April 1). The normal and pathologic renal medulla: A comprehensive overview. Pathology Research and Practice, Vol. 211, pp. 271-280. https://doi.org/10.1016/j. prp.2014.12.009

Mishra, A. K., Manikandan, R., Dorairajan, L. N., Mittal, J. K., \& Rekha, J. S. (2016). Bellini duct carcinoma: A rare entity. Journal of Clinical and Diagnostic Research, 10(10). https://doi. org/10.7860/JCDR/2016/21335.8613

Muglia, V. F., \& Prando, A. (2015). Renal cell carcinoma: histological classification and correlation with imaging findings. Radiologia Brasileira, 48(3), 166174. https://doi.org/10.1590/01003984.2013.1927

Petejova, N., \& Martinek, A. (2016). Renal cell carcinoma: Review of etiology, pathophysiology, and risk factors. Biomedical Papers, Vol. 160, pp. 


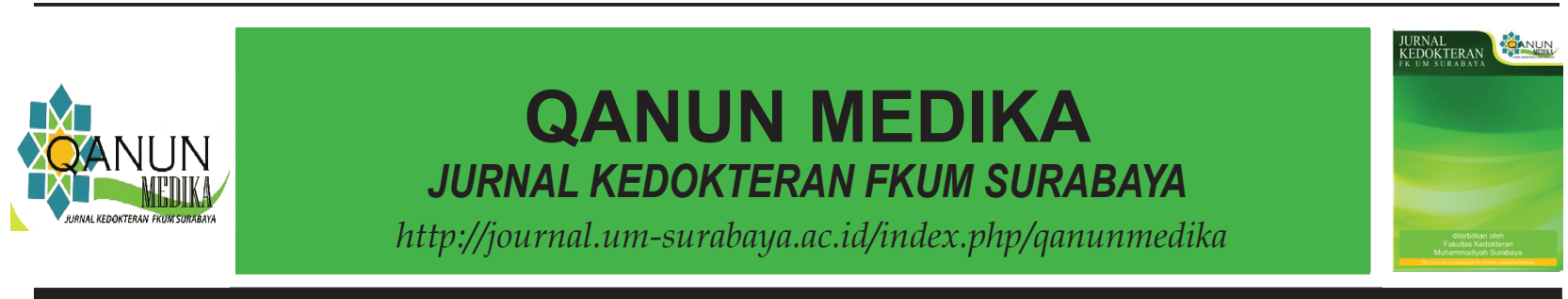

183-194.https://doi.org/10.5507/bp.2015.050

Seo, A. N., Yoon, G., \& Ro, J. Y. (2017). Clinicopathologic and molecular pathology of collecting duct carcinoma and related renal cell carcinomas. Advances in Anatomic Pathology, Vol. 24, pp. 65-77. https://doi.org/10.1097/ PAP.0000000000000138

Shuch, B., Amin, A., Armstrong, A. J., Eble, J. N., Ficarra, V., Lopez-Beltran, A., ... Kutikov, A. (2015, January 1). Understanding pathologic variants of renal cell carcinoma: Distilling therapeutic opportunities from biologic complexity. European Urology, Vol. 67, pp. 85-97. https://doi.org/10.1016/j. eururo.2014.04.029

Sui, W., Matulay, J. T., Robins, D. J., James, M. B., Onyeji, I. C., Roy Choudhury, A., ... DeCastro, G. J. (2017). Collecting duct carcinoma of the kidney: Disease characteristics and treatment outcomes from the National Cancer Database. Urologic Oncology: Seminars and
Original Investigations, 35(9), 540. e13-540.e18. https://doi.org/10.1016/j. urolonc.2017.04.010

Wu, J., Zhu, Q., Zhu, W., Chen, W., \& Wang, S. (2015). Comparative study of CT appearances in mucinous tubular and spindle cell carcinoma and collecting duct carcinoma of the kidney. British Journal of Radiology, 88(1056). https:// doi.org/10.1259/bjr.20140434

Zheng, I., Alameddine, M., Tan, Y., Moghadamyeghaneh, Z., Jue, J. S., Yusufali, A., ... Ciancio, G. (2017). Collecting Duct Carcinoma of the Native Kidney in a Renal Transplant Recipient. CaseReportsinTransplantation, 2017, 1-6. https://doi.org/10.1155/2017/4527104 